Brain, Behavior and Evolution
Brain Behav Evol 2017;89:185-194

DOI: $10.1159 / 000468974$
Received: January 9, 2017

Returned for revision: January 30, 2017

Accepted after revision: March 7, 2017

Published online: May 6, 2017

\title{
Effects of Isometric Brain-Body Size Scaling on the Complexity of Monoaminergic Neurons in a Minute Parasitic Wasp
}

\author{
Emma van der Woude Hans M. Smid \\ Laboratory of Entomology, Wageningen University, Wageningen, The Netherlands
}

\section{Keywords}

Octopamine - Dopamine $\cdot$ Serotonin $\cdot$ Hymenoptera .

Allometry · Confocal laser scanning microscopy

\begin{abstract}
Trichogramma evanescens parasitic wasps show large phenotypic plasticity in brain and body size, resulting in a 5-fold difference in brain volume among genetically identical sister wasps. Brain volume scales linearly with body volume in these wasps. This isometric brain scaling forms an exception to Haller's rule, which states that small animals have relatively larger brains than large animals. The large plasticity in brain size may be facilitated by plasticity in neuron size, in the number of neurons, or both. Here, we investigated whether brain isometry requires plasticity in the number and size of monoaminergic neurons that express serotonin (5HT), octopamine (OA), and dopamine (DA). Genetically identical small and large $T$. evanescens appear to have the same number of 5HT-, OA-, and DA-like immunoreactive cell bodies in their brains, but these cell bodies differ in diameter. This indicates that brain isometry can be facilitated by plasticity in the size of monoaminergic neurons, rather than plasticity in numbers of monoaminergic neurons. Selection pressures on body miniaturization may have resulted in the evolution of miniaturized neural pathways that allow even the smallest wasps to find suitable hosts. Plasticity in the size of neural
\end{abstract}

\begin{tabular}{ll}
\hline KARGER & $\begin{array}{l}\text { ( } 2017 \text { The Author(s) } \\
\text { Published by S. Karger AG, Basel Openger }\end{array}$ \\
E-Mail karger@karger.com & \\
www.karger.com/bbe & This article is licensed under the Creative Commons Attribution- \\
& $\begin{array}{l}\text { NonCommercial-NoDerivatives 4.0 International License (CC BY- } \\
\text { NC-ND) (http://www.karger.com/Services/OpenAccessLicense). } \\
\text { Usage and distribution for commercial purposes as well as any dis- } \\
\text { tribution of modified material requires written permission. }\end{array}$
\end{tabular}

components may be among the mechanisms that underlie isometric brain scaling while maintaining cognitive abilities in the smallest individuals.

๑ 2017 The Author(s)
Published by S. Karger AG, Basel

\section{Introduction}

According to Haller's rule, small animals have relatively larger brains than large animals. This negative allometric brain-body size relationship follows a power law function in which the exponent (the scaling coefficient) describes how mass or volume of the brain scales with mass or volume of the body. This scaling coefficient is smaller than 1 in the case of negative allometry. Haller's rule applies to most animal species studied so far, both in comparisons between species and within species [Wehner et al., 2007; Isler et al., 2008; Seid et al., 2011]. The computational power of a brain may depend more on the absolute number of neurons and connections than on relative brain size [Chittka and Niven, 2009]. Hence, the relatively larger brains in small animals may be the consequence of a need to maintain cognitive abilities, and therefore absolute brain size, in smaller-bodied animals.

Because brain tissue has a high metabolic rate [Aiello and Wheeler, 1995], smaller animals suffer relatively higher energetic costs of maintaining a relatively large

Emma van der Woude

Laboratory of Entomology, Wageningen University PO Box 16

NL-6700 AA Wageningen (The Netherlands)

E-Mail emmavanderwoude@gmail.com 
brain. These increasing energetic demands eventually limit evolutionary miniaturization of a species [Eberhard and Wcislo, 2011], and may similarly limit the variation in body size within a species. Interestingly, some of the smallest animals on Earth appear to "evade" Haller's rule [Van der Woude et al., 2013; Groothuis and Smid, 2017]. Among these are Trichogramma evanescens, minute parasitoid wasps that develop inside the eggs of butterflies and moths. Adult body size depends on the size of the lepidopteran host egg, and on the number of competing parasitoid larvae that develop inside the same egg. This results in phenotypic plasticity in body size; body length can range between 0.3 and $0.9 \mathrm{~mm}$ in genetically identical sister wasps [Van der Woude and Smid, 2016]. T. evanescens parasitic wasps do not show negative brain-body size allometry, but scale their brains in a linear way with body size [Van der Woude et al., 2013]. Such isometric brain scaling, with scaling coefficients equal to 1 , results in the same relative brain size in small and large wasps. This brain-scaling strategy may be an adaptation that allows body miniaturization beyond the limits that are imposed by the energetic trade-offs of allometric brain scaling.

As a consequence of isometric brain scaling, small $T$. evanescens have brains that are smaller than predicted by Haller's rule, and large T. evanescens have brains that are larger than predicted by Haller's rule. Brain size plasticity may, therefore, be more extreme in T. evanescens than in species that scale their brains allometrically. We have previously found a 5 -fold difference in brain volume between genetically similar small and large sister wasps [Van der Woude et al., 2013]. This indicates that there is extreme phenotypic plasticity in brain size in this species, which is solely determined by the amount of nutrition that was available during development. Such extreme plasticity in brain size could be regulated by plasticity in the morphology of neuronal pathways. This may show as differences between small and large wasps in neuron size, in the number of neurons and arborisations, or both. These differences may have functional consequences, because cell bodies [Gregory, 2001] and axons [Faisal et al., 2005; Perge et al., 2012] need a minimum size for adequate functioning, and neural pathways with lower numbers of neurons and connections may have reduced computational power [Chittka and Niven, 2009; Niven and Farris, 2012]. Hence, a trade-off of isometric brain scaling may be that neuron size or number fall beyond functional limits in the smallest brains.

We have previously shown that small and large T. evanescens show a similar level of complexity in the gross morphology of their olfactory system [Van der Woude and Smid, 2016]. Small and large wasps form the same number of glomeruli in the antennal lobe, and the same number of most types of olfactory sensilla on the antennae. The wasps do adapt the size of glomeruli; absolute and relative glomerular volumes were smaller in small wasps. Interestingly, there were no differences between small and large wasps on a cognitive level, shown by similar levels of olfactory and visual memory retention [unpubl. observations]. These findings suggest that despite their isometric brain scaling, T. evanescens are adapted to small body sizes, without apparent compromises to neural functioning. This could be achieved through maintained numbers of neurons, which are not reduced beyond their minimum size for adequate functioning.

Here, we studied how isometric brain scaling affects the number and size of neuronal cell bodies, in 3 quantifiable subsets that express serotonin (5HT), octopamine (OA), and dopamine (DA), respectively. These monoamines are derivatives of amino acids that act as neurotransmitters, neuromodulators, and neurohormones in the insect brain [Roeder, 1994; Libersat and Pflueger, 2004]. They play critical roles in basic neural functioning and are involved in many vital behavioral, cognitive, and physiological processes [Roeder, 1994; Blenau and Thamm, 2011; Burke et al., 2012; Yamamoto and Seto, 2014]. Although the monoaminergic systems may be highly conserved to maintain vital functions, plasticity in neuron numbers could to some extent be possible. This has been shown in Pheidole dentata ants, where the number of serotonergic cell bodies in the optic lobe differs with age and subcaste [Seid et al., 2008]. Similar plasticity in number of neurons could also underlie isometric brain scaling in T. evanescens.

We have previously described the general morphology of 5HT-, DA-, and OA-like immunoreactive (5HT-L-IR, OA-L-IR, and DA-L-IR) neurons in the species T. evanescens, and compared our descriptions to those of larger insects [Van der Woude and Smid, 2017]. The aim of the present study was to unravel whether brain isometry requires plasticity in the size and number of 5HT-L-IR, OA-L-IR, and DA-L-IR neurons in small and large T. evanescens. We focus on phenotypic plasticity in body size in the context of adult body size variation that results from differences in scramble competition during larval development. Hence, we studied genetically identical wasps of similar age and experience, but with large differences in body and brain size. We expected that neuron size is reduced to a functional limit in small wasps, while neurons are larger in large wasps. Furthermore, we ex-
186

Brain Behav Evol 2017;89:185-194 DOI: $10.1159 / 000468974$ van der Woude/Smid 
pected that small wasps show a lower number of monoaminergic neurons in their brains compared to large wasps, to facilitate isometric brain scaling.

\section{Materials and Methods}

\section{Insects}

We used female T. evanescens Westwood (Hymenoptera: Trichogrammatidae) of inbred isofemale strain GD011. Wasps were reared in a climate room $\left(22 \pm 1^{\circ} \mathrm{C}, 50-70 \% \mathrm{rh}, \mathrm{L} 16: \mathrm{D} 8\right)$ in eggs of 3 host species: the Mediterranean flour moth Ephestia kuehniella, the cabbage moth Mamestra brassicae, and the tobacco hornworm Manduca sexta. Eggs of E. kuehniella were obtained as UV-irradiated eggs from Koppert Biological Systems (Berkel en Rodenrijs, The Netherlands). M. brassicae were reared on cabbage plants (Brassica oleracea) in a climate room $(21 \pm$ $\left.2{ }^{\circ} \mathrm{C}, 50-70 \% \mathrm{rh}, \mathrm{L16}: \mathrm{D} 8\right)$. Adult moths oviposited on sheets of filter paper, and their eggs were used fresh for rearing procedures. M. sexta were obtained as pupae from the Max Planck Institute for Chemical Ecology (Jena, Germany). Adults were kept in a flight cage with a tobacco plant (Nicotiana tabacum SR1) inside a climate cabinet $\left(25 \pm 1^{\circ} \mathrm{C}, \mathrm{L} 16: \mathrm{D} 8\right)$. Eggs were collected from the tobacco plants and frozen until use in rearing procedures.

\section{Generating Body Size Variation}

We used 3 species of differently sized host eggs to generate body size variation in T. evanescens. Adult body size depends on the amount of nutrition that was available during larval development and, therefore, on the size of the host egg and the number of developing larvae inside it [Van der Woude et al., 2013; Van der Woude and Smid, 2016]. E. kuehniella eggs are shaped as prolate spheroids of $\sim 0.52 \mathrm{~mm}$ long and $\sim 0.38 \mathrm{~mm}$ in diameter, and with a volume of approximately $0.038 \mathrm{~mm}^{3}$. These eggs can only host a single (occasionally 2 ) developing T. evanescens larvae. Wasps that emerge from these eggs are always small: $0.3-0.4 \mathrm{~mm}$ measured from thorax to the tip of the abdomen [Van der Woude et al., 2013]. M. brassicae eggs are spheroids, with a diameter of $\sim 0.60$ $\mathrm{mm}$ and a volume of $\sim 0.11 \mathrm{~mm}^{3}$. These eggs can support the development of 1-5 wasps, resulting in adults with thorax-abdomen length ranging between 0.3-0.6 mm [Van der Woude et al., 2013]. $M$. sexta eggs are spheroids of approximately $1.40 \mathrm{~mm}$ in diameter and $\sim 1.44 \mathrm{~mm}^{3}$ in volume. These eggs can host 6-40 developing wasps, and adults that emerge from these eggs reach thorax-abdomen lengths of $0.4-0.8 \mathrm{~mm}$ respectively [Van der Woude and Smid, 2016].

To increase the proportion of large wasps that emerged from $M$. brassicae eggs, we observed oviposition behavior on these hosts and removed the wasp after laying her first egg as described previously [Suzuki et al., 1984; Van der Woude et al., 2013]. Host eggs on which oviposition behavior was observed were combined with eggs to which the wasps had unlimited access for $4 \mathrm{~h}$, to ensure that both large and small offspring emerged.

To increase the proportion of large wasps that emerged from $M$. sexta eggs, we manipulated female wasps to lay fewer eggs in these hosts. Drilling through the larger $M$. sexta eggs takes the wasps a long time, and is generally followed by a period of host feeding. This makes these eggs unsuitable for observation of ovi- position behavior. We instead exploited the wasps' host examination behavior to generate body size variation on M. sexta. Wasps of the genus Trichogramma assess host egg size through antennal drumming of its surface, and adapt the number of eggs they lay inside this perceived volume. When the egg appears smaller due to a partially inaccessible surface, Trichogramma wasps lay fewer eggs inside [Schmidt and Smith, 1985; 1987]. Hence, we partially masked the surface of $M$. sexta eggs by distributing them on 5-10 $\mathrm{mL}$ cooling $1 \%$ agarose (Sigma) in a petridish (Greiner Bio-One, $94 \times 15 \mathrm{~mm}$ ) as described before [Van der Woude and Smid, 2016]. This resulted in some eggs being fully exposed for antennal drumming, and some eggs being partially covered by agarose. The wasps had access to these eggs for $4 \mathrm{~h}$. This combination of partially masked and fully exposed $M$. sexta eggs ensured that both small and large wasps emerged.

\section{Analysis of Immunoreactivity}

We prepared the samples as described in Van der Woude and Smid [2017]. In brief, heads of 2-day-old female T. evanescens wasps were partially opened and fixed. Either the anterior or posterior cuticle was removed with fine tweezers to allow imaging of the brain. These heads were stained with either rabbit anti5HT antibodies (Millipore Cat\# AB938, RRID:AB_92263), rabbit anti-OA antibodies (MoBiTec Cat\# 1003GE, RRID:AB_2314999) or mouse anti-DA antibodies (Millipore Cat\# MAB5300, RRID:AB_94817), all at a 1:200 dilution. For analysis of OA and 5HT, a secondary antiserum of goat-antirabbit antibodies linked to Alexa fluor 488 (Molecular Probes, Cat\# A11008, RRID:AB_143165) was applied at a 1:200 dilution together with 1:500 propidium iodide (Sigma-Aldrich). For analysis of DA, a secondary antibody of rabbit-anti-mouse (Dako Cat\# Z0259, RRID:AB_2532147) was applied at a 1:200 dilution, followed by a tertiary antiserum of goat-anti-rabbit antibodies linked to Alexa Fluor 488 (Jackson ImmunoResearch Labs Cat\# 115-545-003, RRID:AB_2338840) at a 1:200 dilution together with 1:500 propidium iodide. Heads were scanned with a Zeiss LSM 510 confocal laser scanning microscope, using a 488-nm argon laser and a Plan-Apochromat $\times 63$ oil immersion objective (N.A. 1.4). A band pass emission filter at 505-550 nm was used to visualize Alexa Fluor 488, and a long pass emission filter at $560 \mathrm{~nm}$ for propidium iodide.

\section{Classification of Brain Size}

We measured brain width and brain height from optical sections to differentiate between small and large wasps. The tight attachment of the lamina to the compound eye caused damage to this area when the reflective layer had to be removed for imaging. We therefore used the lateral boundaries of the medullas as proxy for brain width. The distance between the dorsal and ventral rim of the brain was used to estimate brain height. Measurements were performed in optical cross sections using the measurement tool in the Fiji package of ImageJ 1.48s [Schindelin et al., 2012]. These distances were ranked and used to select the 15 largest and 15 smallest well-stained brains of each antibody treatment.

\section{Neuron Analysis}

Image segmentation was used to accurately determine the number of neurons in clusters that contained many cells close together. Cell bodies of paired neuron clusters (all clusters except 


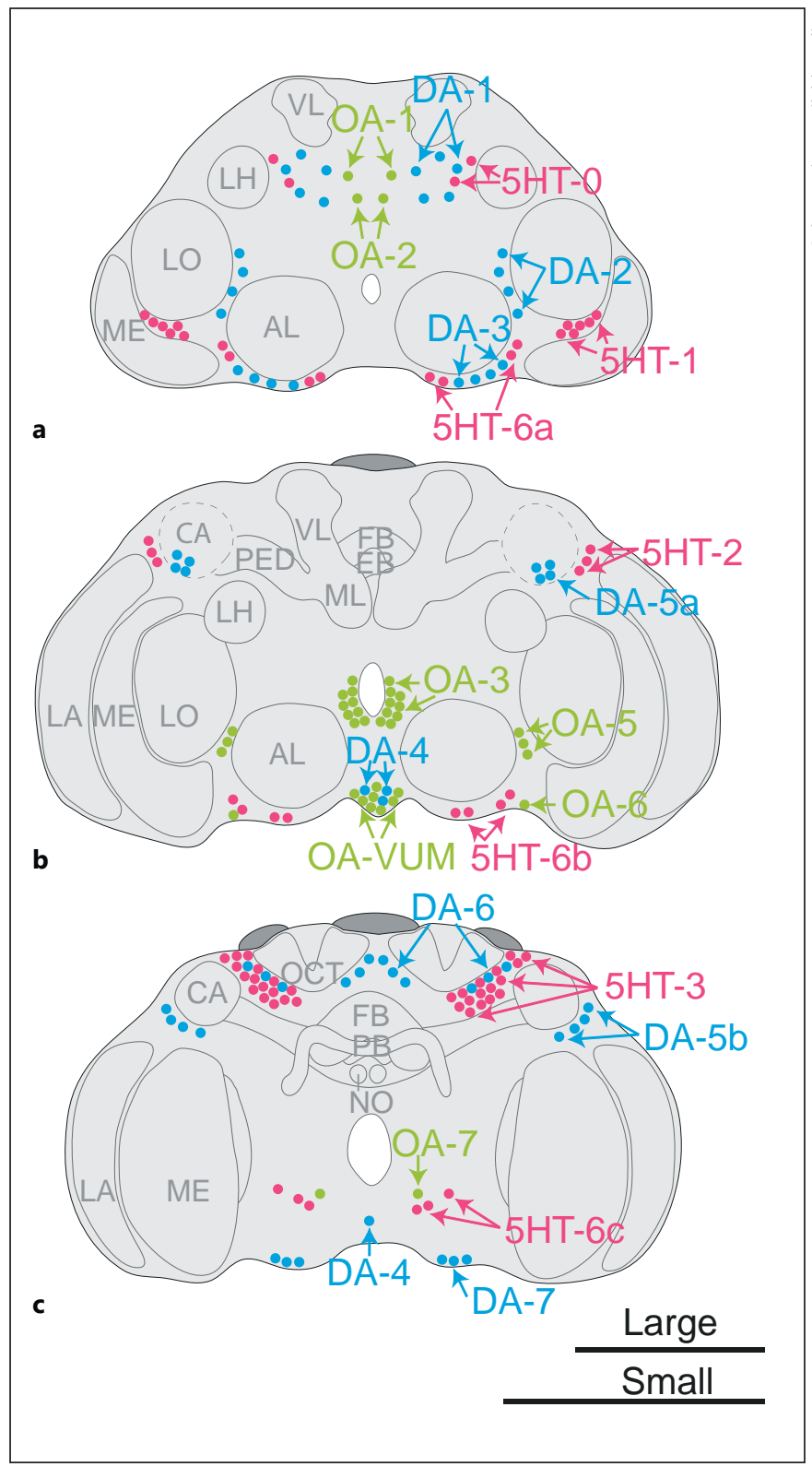

Fig. 1. Overview of distribution of serotonin (5HT)-, octopamine (OA)-, and dopamine (DA)-like immunoreactive neuron clusters in the brain of T. evanescens. a Anterior view with dorsal side up at approximately one-quarter in depth of the brain. b Anterior view halfway in depth of the brain. Dashed lines indicate the outline of the calyx, which is located posterior to cluster DA-5a. c Posterior view 3 quarters in depth of the brain. Shown cell body distributions are combined from separate experiments for the 3 monoamines [Van der Woude and Smid, 2017], hence the relative locations of different monoaminergic clusters may differ from this schematic representation. AL, antennal lobe; LA, lamina; ME, medulla; LO, lobula; LH, lateral horn; CA, calyx; PED, pedunculus; VL, vertical lobe; ML, medial lobe; FB, fan-shaped body; EB, ellipsoid body; PB, protocerebral bridge; NO, noduli; OCT, ocellar tract. Scale bars indicate $50 \mu \mathrm{m}$, top scale bar for an average large brain, bottom scale bar for an average small brain.
OA-VUM and DA-4) were analyzed in the best stained hemisphere of each selected brain. We used either the segmentation editor of Amira 5.4 (Visage Imaging $\mathrm{GmbH}$, Berlin, Germany) or the TrakEM2 plugin [Cardona et al., 2012] in the Fiji package of ImageJ 1.48s [Schindelin et al., 2012]. Cell diameters were measured with the measuring tool in the Fiji package of ImageJ 1.48s. Each cell body was measured twice, and measurements of all cells within a cluster were averaged to obtain a single average value per cluster per brain.

\section{Statistical Analysis}

Differences in brain width and height between small and large wasps were analyzed with independent samples $t$ tests. Generalized linear models with Poisson distribution and log-link function were used to analyze the number of cell bodies in neuron clusters, using size class and neuron cluster as fixed factors. Type III Wald $\chi^{2}$ analysis of deviance was used to test for significance of main effects. Cell body diameter was analyzed with linear models, using log-transformed diameters to obtain normally distributed residuals. Analysis of variance was used to test for significance of main effects, followed by Tukey HSD post-hoc tests. In all cases, separate models were run for the separate monoamine experiments. All analyses were performed in $\mathrm{R}$ version 3.1 .0 at alpha $=0.05$. Values are shown as mean \pm SD.

\section{Results}

\section{Immunolabelling}

The antisera that we used provided clear immunolabelling results, but there was a difference in the intensity of staining between the 3 antisera. The 5HT-L-IR staining was more intense than OA- and DA-L-IR staining. There appeared to be no differences in intensity of the immunoreactive staining between differentially sized brains. All detected neuron clusters were visible in wasps of all sizes (Fig. 1). An elaborate description of the precise morphology and location of neuron clusters, also in comparison to other insects, can be found elsewhere [Van der Woude and Smid, 2017].

\section{Brain Size Variation}

We measured the size of the brains in the small and large size classes for each of the 3 separate monoamine immunolabelling treatments $(n=15$ for each combination of size class and monoamine treatment). Brain size was measured from left to right medulla, and from dorsal to ventral rim of the brain (Table 1; Fig. 2). Small wasps had significantly smaller brain widths (5HT: $\mathrm{t}_{25}=23.390$, $p<0.001$; OA: $\mathrm{t}_{23}=10.156, p<0.001$; DA: $\mathrm{t}_{24}=10.028$, $p<0.001)$ and brain heights (5HT: $\mathrm{t}_{27}=12.572, p<0.001$; OA: $\left.\mathrm{t}_{23}=12.632, p<0.001 ; \mathrm{DA}: \mathrm{t}_{22}=6.354, p<0.001\right)$ than large wasps. 


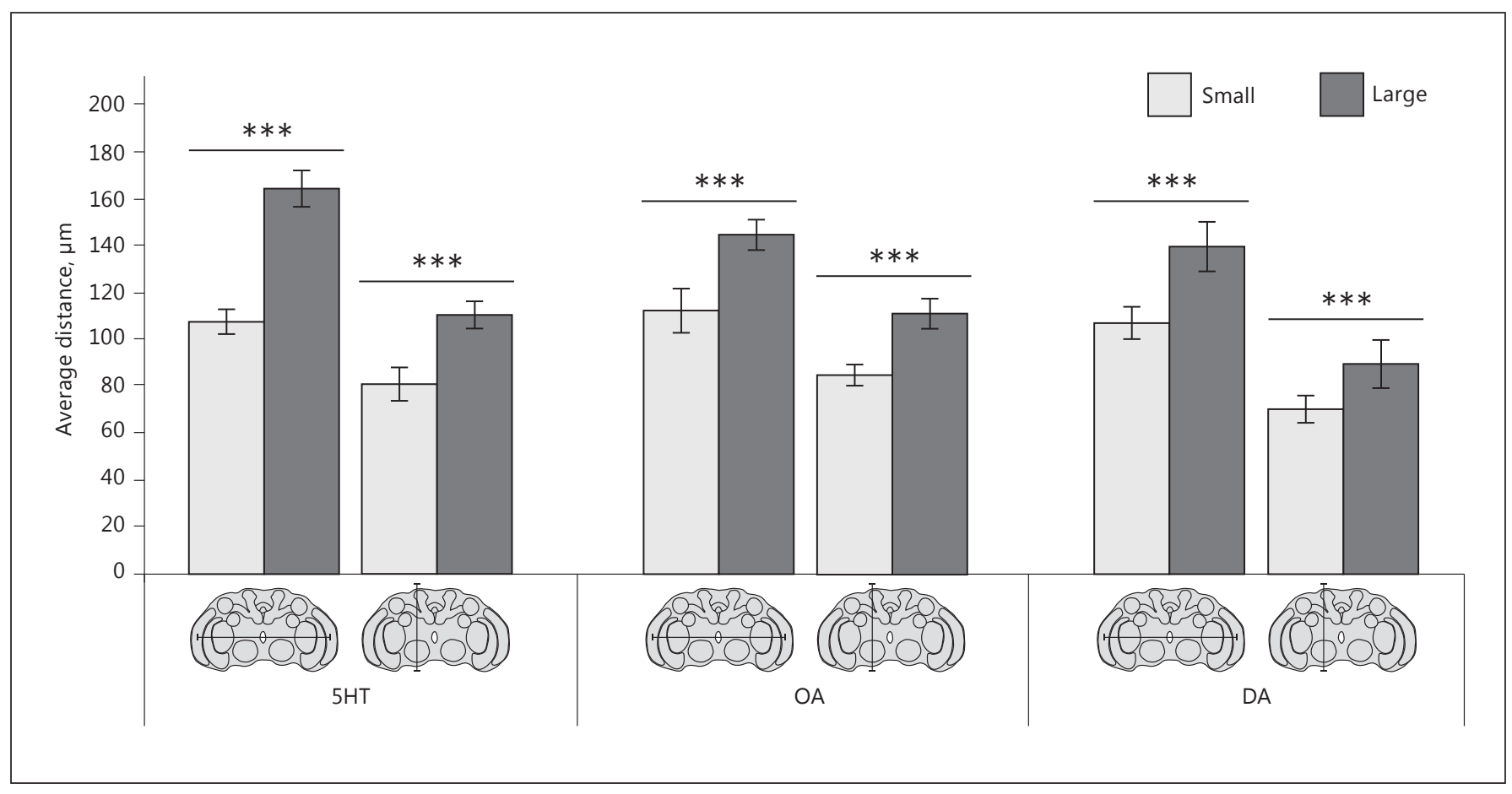

Fig. 2. Difference in brain size between the small and large wasps that were used to analyze serotonin (5HT)-, octopamine (OA)-, and dopamine (DA)-like immunoreactive neurons, showing the average distance $( \pm S D)$ measured from left to right medullas and from dorsal to ventral rim of the brain, as indicated in the corres- ponding graphs below the bars. Brain size was measured of 15 wasps per size class and monoamine treatment. Asterisks indicate significant differences in brain width or height between small and large wasps based on independent samples $t$ tests; ${ }^{* * *} p<0.001$.

Table 1. Average brain width and height $( \pm \mathrm{SD})$ of small and large wasps that were used to analyze serotonin $(5 \mathrm{HT})-$, octopamine $(\mathrm{OA})-$, and dopamine (DA)-like immunoreactive neurons

\begin{tabular}{|c|c|c|c|c|c|c|}
\hline & \multicolumn{3}{|c|}{ Brain width $\pm \mathrm{SD}, \mu \mathrm{m}$} & \multicolumn{3}{|c|}{ Brain height $\pm \mathrm{SD}, \mu \mathrm{m}$} \\
\hline & small & large & $\begin{array}{l}\text { difference, } \\
p \text { value }\end{array}$ & small & large & $\begin{array}{l}\text { difference, } \\
p \text { value }\end{array}$ \\
\hline $5 \mathrm{HT}$ & $107 \pm 5.29(n=15)$ & $164 \pm 7.75(n=15)$ & $<0.001$ & $81 \pm 7.13(n=15)$ & $110 \pm 5.79(n=15)$ & $<0.001$ \\
\hline $\mathrm{OA}$ & $112 \pm 9.45(n=15)$ & $145 \pm 6.45(n=15)$ & $<0.001$ & $85 \pm 4.49(n=15)$ & $111 \pm 6.43(n=15)$ & $<0.001$ \\
\hline DA & $107 \pm 6.85(n=15)$ & $140 \pm 10.54(n=15)$ & $<0.001$ & $70 \pm 5.78(n=15)$ & $89 \pm 10.18(n=15)$ & $<0.001$ \\
\hline
\end{tabular}

Differences in brain width and height between small and large wasps are based on independent samples $t$ tests.

\section{Effects of Body Size on Number of Cell Bodies}

Generalized linear models revealed that there was no difference in number of monoaminergic cell bodies between small and large wasps $\left(5 \mathrm{HT}: \chi^{2}{ }_{1}=2.979, p=0.084\right.$; OA: $\chi^{2}{ }_{1}=2.897, p=0.089$; DA: $\chi^{2}{ }_{1}=1.450, p=0.229$; Fig. 3; Table 2). We counted on average $20.8 \pm 5.24(n=$ 30) 5HT-L-IR cell bodies per brain, $9.03 \pm 4.52(n=30)$ OA-L-IR cell bodies, and $14.7 \pm 5.31(n=30)$ DA-L-IR cell bodies. There was a difference in the number of cell bodies between the different clusters (5HT: $\chi^{2}{ }_{6}=420.791$, $p<0.001$; OA: $\chi^{2}{ }_{6}=73.034, p<0.001 ; \mathrm{DA}: \chi_{6}{ }_{6}=23.553$, $p<0.001)$. Most cell bodies were observed in cluster $5 \mathrm{HT}-3$, which contained up to 16 cell bodies (on average $12.1 \pm 1.70)$. Many OA-L-IR neuron clusters contained only a single pair of cell bodies in all preparations, but cluster OA-VUM contained up to 13 cell bodies. How- 


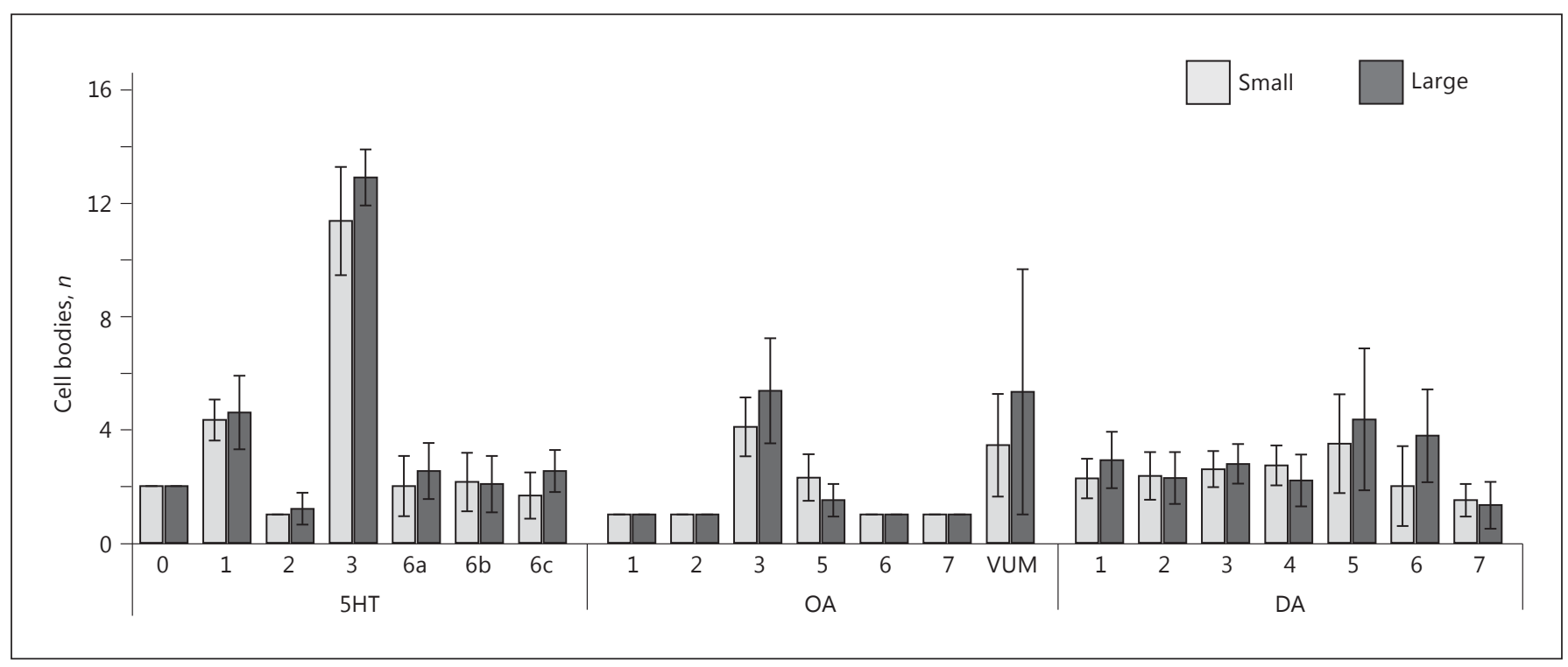

Fig. 3. Average number of cell bodies $( \pm \mathrm{SD})$ per cluster of serotonin $(5 \mathrm{HT})$-, octopamine (OA)-, and dopamine (DA)-like immunoreactive neurons in small and large brains of T. evanescens. There was no effect of brain size on the number of cell bodies. $n$ values are shown in Table 2.

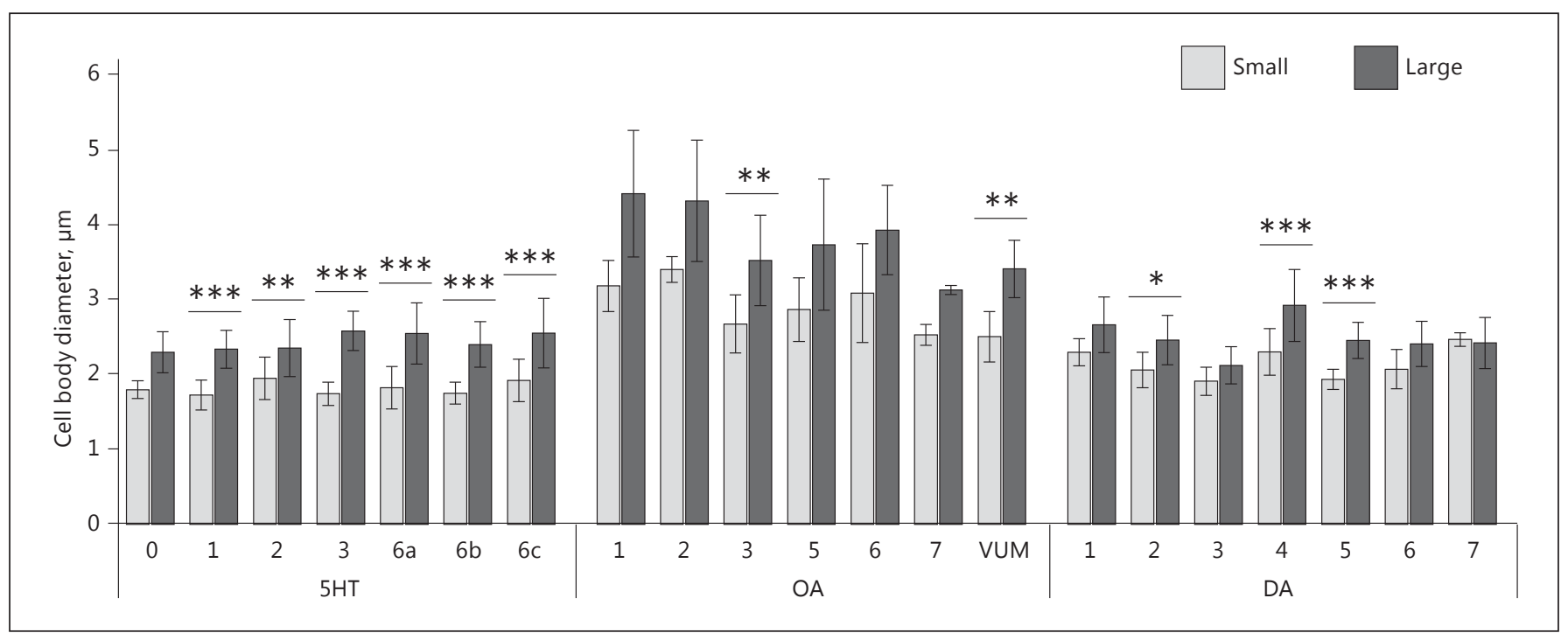

Fig. 4. Average diameter $( \pm \mathrm{SD}$ ) of cell bodies per cluster of serotonin (5HT)-, octopamine (OA)-, and dopamine (DA)-like immunoreactive neurons in small and large brains of T. evanescens. $n$ values are shown in Table 2. Asterisks indicate significant differences in cell body diameter between small and large wasps based on linear models and Tukey HSD post-hoc tests; ${ }^{*} p<0.05,{ }^{* *} p<0.01,{ }^{* * *} p<0.001$.

ever, the close location of this cluster to the mouthparts was vulnerable to damage during the dissection procedure, which resulted in an average count of $4.4 \pm 3.36$ OA-VUM neurons per brain. Table 2 shows details of the cell body counts for each neuron cluster, separately for small and large wasps, and for small and large wasps combined. The interactions between size class and neuron cluster were not significant $\left(5 \mathrm{HT}: \chi^{2}{ }_{6}=2.073, p=\right.$ 0.913; OA: $\chi_{6}^{2}=3.351, p=0.764$; DA: $\chi^{2}{ }_{6}=4.527, p=$ $0.606)$. 
Table 2. Number and diameter of cell bodies of serotonin- (5HT), octopamine (OA)-, and dopamine (OA)-like immunoreactive neurons in the brains of small and large T. evanescens

\begin{tabular}{|c|c|c|c|c|c|c|c|c|c|c|c|c|c|}
\hline & \multicolumn{6}{|l|}{ Count } & & \multicolumn{6}{|l|}{ Diameter } \\
\hline & $\begin{array}{l}\text { maxi- } \\
\text { mum }\end{array}$ & small & $n_{\text {small }}$ & large & $n_{\text {large }}$ & average & & small & $n_{\text {small }}$ & large & $n_{\text {large }}$ & $\begin{array}{l}\text { difference, } \\
p \text { value }\end{array}$ & average \\
\hline \multicolumn{14}{|l|}{$5 H T$} \\
\hline $5 \mathrm{HT}-0$ & 2 & 2.0 & 3 & 2.0 & 3 & 2.0 & $5 \mathrm{HT}-0$ & $1.8 \pm 0.12$ & 3 & $2.3 \pm 0.27$ & 3 & 0.581 & $2.0 \pm 0.33$ \\
\hline $5 \mathrm{HT}-1$ & 6 & $4.3 \pm 0.72$ & 15 & $4.6 \pm 1.30$ & 15 & $4.5 \pm 1.04$ & $5 \mathrm{HT}-1$ & $1.7 \pm 0.20$ & 15 & $2.3 \pm 0.25$ & 15 & $<0.001$ & $2.0 \pm 0.38$ \\
\hline $5 \mathrm{HT}-2$ & 3 & 1.0 & 15 & $1.2 \pm 0.56$ & 15 & $1.1 \pm 0.40$ & $5 \mathrm{HT}-2$ & $1.9 \pm 0.28$ & 15 & $2.3 \pm 0.38$ & 15 & 0.007 & $2.1 \pm 0.39$ \\
\hline $5 \mathrm{HT}-3$ & 16 & $11.4 \pm 1.91$ & 10 & $12.9 \pm 0.99$ & 11 & $12.1 \pm 1.70$ & $5 \mathrm{HT}-3$ & $1.7 \pm 0.16$ & 10 & $2.6 \pm 0.26$ & 11 & $<0.001$ & $2.1 \pm 0.48$ \\
\hline $5 \mathrm{HT}-6 \mathrm{a}$ & 4 & $2.0 \pm 1.07$ & 15 & $2.5 \pm 0.99$ & 15 & $2.3 \pm 1.05$ & 5НТ-6а & $1.8 \pm 0.28$ & 15 & $2.5 \pm 0.41$ & 15 & $<0.001$ & $2.2 \pm 0.48$ \\
\hline 5HT-6b & 4 & $2.1 \pm 1.03$ & 15 & $2.1 \pm 1.00$ & 15 & $2.1 \pm 0.99$ & $5 \mathrm{HT}-6 \mathrm{~b}$ & $1.7 \pm 0.15$ & 15 & $2.4 \pm 0.30$ & 15 & $<0.001$ & $2.1 \pm 0.41$ \\
\hline $5 \mathrm{HT}-6 \mathrm{c}$ & 3 & $1.7 \pm 0.82$ & 14 & $2.5 \pm 0.74$ & 14 & $2.1 \pm 0.89$ & $5 \mathrm{HT}-6 \mathrm{c}$ & $1.9 \pm 0.29$ & 14 & $2.5 \pm 0.46$ & 14 & $<0.001$ & $2.2 \pm 0.50$ \\
\hline \multicolumn{14}{|l|}{$O A$} \\
\hline OA-1 & 1 & 1.0 & 4 & 1.0 & 5 & 1.0 & OA-1 & $3.2 \pm 0.34$ & 4 & $4.4 \pm 0.85$ & 5 & 0.101 & $3.9 \pm 0.91$ \\
\hline OA-2 & 1 & 1.0 & 4 & 1.0 & 5 & 1.0 & OA-2 & $3.4 \pm 0.17$ & 4 & $4.3 \pm 0.81$ & 5 & 0.576 & $3.9 \pm 0.76$ \\
\hline OA-3 & 9 & $4.1 \pm 1.04$ & 11 & $5.4 \pm 1.86$ & 11 & $4.7 \pm 1.61$ & OA-3 & $2.7 \pm 0.39$ & 11 & $3.5 \pm 0.60$ & 11 & 0.006 & $3.1 \pm 0.66$ \\
\hline OA-5 & 3 & $2.3 \pm 0.82$ & 10 & $1.5 \pm 0.58$ & 4 & $2.1 \pm 0.83$ & OA-5 & $2.9 \pm 0.42$ & 10 & $3.7 \pm 0.87$ & 4 & 0.200 & $3.1 \pm 0.68$ \\
\hline OA-6 & 1 & 1.0 & 6 & 1.0 & 5 & 1.0 & OA-6 & $3.1 \pm 0.66$ & 6 & $3.9 \pm 0.60$ & 5 & 0.264 & $3.5 \pm 0.74$ \\
\hline OA-7 & 1 & 1.0 & 3 & 1.0 & 2 & 1.0 & OA-7 & $2.5 \pm 0.14$ & 3 & $3.1 \pm 0.06$ & 2 & 0.949 & $2.8 \pm 0.35$ \\
\hline OA-VUM & 13 & $3.4 \pm 1.81$ & 9 & $5.3 \pm 4.33$ & 9 & $4.4 \pm 3.36$ & OA-VUM & $2.5 \pm 0.34$ & 9 & $3.4 \pm 0.38$ & 9 & 0.003 & $3.0 \pm 0.58$ \\
\hline \multicolumn{14}{|l|}{$D A$} \\
\hline DA-1 & 5 & $2.3 \pm 0.70$ & 15 & $2.9 \pm 1.10$ & 12 & $2.6 \pm 0.89$ & DA-1 & $2.3 \pm 0.18$ & 15 & $2.7 \pm 0.37$ & 12 & 0.134 & $2.5 \pm 0.33$ \\
\hline DA-2 & 4 & $2.4 \pm 0.84$ & 14 & $2.3 \pm 0.91$ & 14 & $2.3 \pm 0.86$ & DA-2 & $2.1 \pm 0.24$ & 14 & $2.5 \pm 0.33$ & 14 & 0.011 & $2.3 \pm 0.35$ \\
\hline DA-3 & 4 & $2.6 \pm 0.63$ & 15 & $2.8 \pm 0.70$ & 14 & $2.7 \pm 0.66$ & DA-3 & $1.9 \pm 0.19$ & 15 & $2.1 \pm 0.25$ & 14 & 0.527 & $2.0 \pm 0.24$ \\
\hline DA-4 & 4 & $2.7 \pm 0.70$ & 15 & $2.2 \pm 0.92$ & 10 & $2.5 \pm 0.82$ & DA-4 & $2.3 \pm 0.31$ & 15 & $2.9 \pm 0.48$ & 10 & $<0.001$ & $2.5 \pm 0.49$ \\
\hline DA-5 & 8 & $3.5 \pm 1.74$ & 14 & $4.4 \pm 2.50$ & 14 & $3.9 \pm 2.16$ & DA-5 & $1.9 \pm 0.13$ & 14 & $2.5 \pm 0.24$ & 14 & $<0.001$ & $2.2 \pm 0.33$ \\
\hline DA-6 & 6 & $2.0 \pm 1.41$ & 4 & $3.8 \pm 1.64$ & 9 & $3.2 \pm 1.74$ & DA-6 & $2.1 \pm 0.26$ & 4 & $2.4 \pm 0.30$ & 9 & 0.704 & $2.3 \pm 0.32$ \\
\hline DA-7 & 3 & $1.5 \pm 0.58$ & 4 & $1.3 \pm 0.82$ & 6 & $1.4 \pm 0.70$ & DA-7 & $2.5 \pm 0.09$ & 4 & $2.4 \pm 0.34$ & 6 & 1.000 & $2.4 \pm 0.26$ \\
\hline
\end{tabular}

Cell body counts show the maximum number of cell bodies per cluster, and the average number $( \pm \mathrm{SD})$ in small, large, and all measured wasps. There was no difference in number of cell bodies per clusters between small and large wasps. Average cell body diameters $( \pm S D)$ are shown for small, large, and all measured wasps. Differences in cell body diameter between small and large wasps are based on linear models and Tukey HSD post-hoc tests.

\section{Effects of Body Size on Size of Cell Bodies}

Average diameters of the cell bodies in the different clusters ranged between 1.7 and $4.4 \mu \mathrm{m}$ (Table 2).

Linear models showed that cell body diameter was larger in large wasps than in small wasps (5HT: $\mathrm{F}_{1,161}=221.537$, $p<0.001 ;$ OA: $\mathrm{F}_{1,74}=73.495, p<0.001 ; \mathrm{DA}: \mathrm{F}_{1,146}=65.662$, $p<0.001$; Fig. 4). Average diameter of 5HT-L-IR cell bodies was $1.8 \pm 0.23 \mu \mathrm{m}(n=88)$ for small wasps and $2.4 \pm$ $0.36 \mu \mathrm{m}(n=87)$ for large wasps. Average diameter of OAL-IR cell bodies was $2.8 \pm 0.48 \mu \mathrm{m}(n=47)$ for small wasps and $3.8 \pm 0.71 \mu \mathrm{m}(n=41)$ for large wasps. The average diameter of DA-L-IR cell bodies was $2.1 \pm 0.28 \mu \mathrm{m}(n=$ 81) for small wasps and $2.5 \pm 0.39 \mu \mathrm{m}(n=79)$ for large wasps. Tukey HSD post-hoc tests (Table 2) revealed that cell body diameters differed between small and large wasps for all 5HT-L-IR clusters except cluster 5HT-0. In contrast, the only OA-L-IR and DA-L-IR clusters that differed in diameter between small and large wasps were cluster OA-3, OA-VUM, DA-4, and DA-5.

Brain Isometry Affects Monoaminergic Neurons
Serotonergic cell bodies are similarly sized in all clusters $\left(\mathrm{F}_{6,161}=1.652, p=0.136\right)$, whereas the diameters of octopaminergic $\left(\mathrm{F}_{6,74}=6.065, p<0.001\right)$ and dopaminergic $\left(\mathrm{F}_{6,146}=12.266, p<0.001\right)$ cell bodies vary between different clusters. The interactions between size class and cell body diameter were not significant $\left(5 \mathrm{HT}: \mathrm{F}_{6,161}=\right.$ $1.554, p=0.164 ; \mathrm{OA}: \mathrm{F}_{6,74}=0.164, p=0.986 ; \mathrm{DA}: \mathrm{F}_{6146}=$ $2.1660, p=0.050)$.

\section{Discussion}

Our results show that isometric brain scaling in T. evanescens may be facilitated by plasticity in the size of the cell bodies, rather than in the numbers of monoaminergic neurons. Small and large wasps show no differences in the number of serotonergic, dopaminergic and octopaminergic cell bodies in their brains, but they do show differences in the size of these cell bodies. This suggests that

Brain Behav Evol 2017;89:185-194 191 
monoaminergic neurons support neural and behavioral functions that are vital for even the smallest wasps. Maintaining the number of monoaminergic neurons may maintain cognitive and behavioral complexity, and allow even the smallest wasps to find suitable hosts.

Isometric brain scaling results in brains that are smaller than is predicted by Haller's rule in small T. evanescens, and in brains that are larger than is predicted in large $T$. evanescens. We expected that this extreme brain size plasticity required modifications to the number and size of neurons. Specifically, we hypothesized that plasticity in cell body size alone would not be sufficient to achieve isometric brain scaling, because cell body size may approach functional limits in small wasps. We expected that an additional decrease of neuron number would be required to achieve the smallest brains. Our results show a difference in neuronal cell body size between small and large wasps, but no difference in the number of monoaminergic neurons. This indicates that plasticity in cell-body size, at least in these specific sets of neurons, can be sufficient to achieve isometric brain scaling.

Similar modifications are shown on neuropil level in the antennal lobe. The antennal lobe consists of several spherical glomeruli, which are functional units that contain the synapses of olfactory receptor neurons, projection neurons and interneurons [Hansson and Anton, 2000]. We have previously shown that the number of antennal lobe glomeruli is similar in small and large T. evanescens, but there is plasticity in glomerular volume [Van der Woude and Smid, 2016]. The combined results of the present and previous studies indicate that neural complexity can be similar in small and large wasps, at least on the level of neuropil structures and of individual neurons. However, differences in neural complexity may occur on other levels, such as those of synaptic connections.

The number of neurons inside a brain is an important component of its computational power [Chittka and Niven, 2009]. Hence, the similarities in the numbers of monoaminergic cell bodies and antennal lobe glomeruli in small and large wasps could imply that the function of neuropil structures and neural pathways is also maintained. We have previously investigated memory retention capacities of small and large wasps after a single olfactory or visual conditioning experience [unpubl. observations]. We indeed found that small and large wasps show similar memory retention levels and duration of memory retention. Plasticity in the size of neuronal cell bodies and neuropil structures may be one of the mechanisms that underlie isometric brain scaling and simultaneously maintains cognitive abilities.
Brain Behav Evol 2017;89:185-194 DOI: $10.1159 / 000468974$
Maintained numbers of neurons may be specific for these monoaminergic clusters, or a general effect of isometric brain scaling that is also shown in other neuronal systems. If neuron numbers are only maintained in these specific monoaminergic neuron clusters, this may mean that modifications of these clusters are too costly, for example because they facilitate the high level of behavioral and cognitive complexity that is required to locate suitable host eggs. If neuron numbers are generally maintained in all neuronal systems, this may have evolved as a consequence of selection pressures on body miniaturization. Trichogramma wasps can parasitize and develop inside very small host eggs. Some of the smallest hosts can only support a single developing $T$. evanescens that will develop into a small adult [Salt, 1940]. The limited availability of nutrients in such small hosts may severely constrain development, especially the investment in metabolically expensive tissues such as the brain [Aiello and Wheeler, 1995]. These selection pressures may have resulted in the evolution of miniaturized neural pathways that support the behavioral and cognitive requirements of even the smallest $T$. evanescens wasps. The larger size of neuronal cell bodies of large wasps indicates that there may be costs associated with having small neurons, which outweigh the benefits of increased numbers of neurons. These costs may not be associated with memory retention [unpubl. observations], but can be present in other neural, behavioral or physiological traits.

Although smaller neurons are more energy-efficient than larger neurons [Niven, 2016], there are costs associated with reducing neuron size. The reduction of neural membrane area results in less space for ion channels, which increases neural noise [Niven and Farris, 2012]. Smaller neurons can also have thinner neurites with reduced neural firing rates [Niven, 2016]. They may also have a reduced number of arborizations and a lower level of connectivity between neurons. Reduction of cytoplasm volume may reduce the available space for mitochondria, and may therefore negatively affect the generation of energy [Niven and Farris, 2012]. A reduction of cell body volume may also affect the volume of the cell organelles, of which the nucleus is the largest. A reduction of nucleus size may involve modifications of genome size or chromatin compaction [Gregory, 2001; Polilov, 2015]. These modifications may compromise neural functioning, because they affect transcription dynamics. Even more extreme reductions of cell body size are shown by another minute member of the family Trichogrammatidae, the $0.2 \mathrm{~mm}$ long Megaphragma mymaripenne [Polilov, 2012]. Approximately $95 \%$ of the neural nuclei lyse during pupal

van der Woude/Smid 
development, resulting in an adult brain with mostly anucleate neurons. Lysis of neural nuclei has not been observed in T. evanescens [Polilov, 2016]. The lack of nuclei implies that anucleate neurons are incapable of genetic transcription, which may severely impair neural functioning in adult M. mymaripenne. This may cause reduced longevity of $M$. mymaripenne compared to other wasps of the family Trichogrammatidae, and could explain why honey-fed M. mymaripenne live on average 5 days at a temperature of $25^{\circ} \mathrm{C}$ [Bernardo and Viggiani, 2000], whereas, for example, Trichogramma minutum live on average 25 days at the same temperature [ $\mathrm{Yu}$ et al., 1984].

The costs of having small cell bodies may be similar for small T. evanescens as described above. Small wasps may have cell bodies with a higher level of chromatin compaction, which may hinder transcription, and less space for mitochondria, which may negatively affect the available amount of energy. Although we could not accurately establish this with the present methodology, neurites of small $T$. evanescens may also be reduced in size and in arborization complexity. This can result in lower information processing rates and increased neural noise [Niven, 2016]. These neural modifications could negatively affect fitness, for example by contributing to the reduced longevity of small T. evanescens compared to larger conspecifics [Waage and Ming, 1984; Doyon and Boivin, 2005]. Having larger neurons may be a factor that contributes to higher longevity of large wasps. Whether the ratio between nucleus and cell body size was different in small and large T. evanescens could not be observed in this study, because the separation between the antibodyand propidium iodide-stained channels was not clear enough to accurately measure nucleus size. More detailed TEM studies are required to show if neuronal cell body size is modified through changes to the volume of cytoplasm, the nucleus, or both, and if the size and connectivity of neurites is similarly affected. This may reveal if the lower limit to neuron size is reached in the smallest $T$. evanescens.

In conclusion, the results of this study shed light on how the smallest insects manage to evade Haller's rule. In the monoaminergic systems that we studied, isometric brain scaling appears to be facilitated by modification of neuronal cell body size, whereas neuron numbers are maintained. These modifications resemble those on neuropil level in the antennal lobe, where glomerular volumes differ in small and large wasps, but numbers of glomeruli are maintained [Van der Woude and Smid, 2016]. The absence of changes in the numbers of mono-

Brain Isometry Affects Monoaminergic

Neurons aminergic cell bodies and antennal lobe glomeruli suggests that the performance of neural pathways may be similar in small and large T. evanescens, which corresponds to our findings of similar olfactory and visual memory retention in small and large wasps [unpubl. observations]. Plasticity in the size of neuronal cell bodies may be one of the "tricks" to evade Haller's rule and simultaneously maintain cognitive abilities in the smallest wasps. However, differences in neural complexity between small and large wasps may occur on levels outside the scope of the present study, such as those of synapses, which should be identified in further research.

\section{Acknowledgments}

We thank the Max Planck Institute for Chemical Ecology (Jena, Germany) for providing M. sexta pupae; Léon Westerd, Frans van Aggelen, and André Gidding for culturing M. brassicae; the experimental farm of Wageningen University (Unifarm) for growing the tobacco plants; Norbert de Ruijter (Wageningen University, Laboratory of Cell Biology) and Henk Schipper (Wageningen University, Experimental Zoology) for use of the confocal laser scanning microscopes; and Marcel Dicke for constructive comments on a previous version of this article. This work was supported by NWO PE\&RC Graduate Program grant 022.002.004 (to E.W.) and by NWO Open Competition grant 820.01.012 (to H.M.S.).

\section{Disclosure Statement}

The authors declare that they have no conflict of interest.

References
Aiello L, Wheeler P (1995): The expensive-tissue
hypothesis - the brain and the digestive sys-
tem in human and primate evolution. Curr
Anthropol 36:199-221.
Bernardo U, Viggiani G (2000): Biological data on
Megaphragma amalphitanum Viggiani and
Megaphragma mymaripenne Timberlake
(Hymenoptera: Trichogrammatidae), egg-
parasitoid of H. haemorrhoidalis (Bouché)
(Thysanoptera: Thripidae) in southern Italy.
Boll Lab Entomol Agrar Filippo Silvestri 58:
77-85.
Blenau W, Thamm M (2011): Distribution of se-
rotonin (5-HT) and its receptors in the insect
brain with focus on the mushroom bodies:
lessons from Drosophila melanogaster and
Apis mellifera. Arthropod Struct Dev 40:381-
394.
Burke CJ, Huetteroth W, Owald D, Perisse E,
Krashes MJ, Das G, Gohl D, Silies M, Certel S,
Waddell S (2012): Layered reward signalling
through octopamine and dopamine in Dro-
sophila. Nature 492:433-437.

Brain Behav Evol 2017;89:185-194 DOI: $10.1159 / 000468974$ 
Cardona A, Saalfeld S, Schindelin J, ArgandaCarreras I, Preibisch S, Longair M, Tomancak P, Hartenstein V, Douglas RJ (2012): TrakEM2 software for neural circuit reconstruction. PLoS One 7:e38011.

Chittka L, Niven J (2009): Are bigger brains better? Curr Biol 19:R995-R1008.

Doyon J, Boivin G (2005): The effect of development time on the fitness of female Trichogramma evanescens. J Insect Sci 5:4.

Eberhard WG, Wcislo WT (2011): Grade changes in brain-body allometry: morphological and behavioural correlates of brain size in miniature spiders, insects and other invertebrates; in Casas J (ed): Spider Physiology and Behaviour. Burlington, Academic Press, vol 40, pp $155-214$.

Faisal AA, White JA, Laughlin SB (2005): Ionchannel noise places limits on the miniaturization of the brain's wiring. Curr Biol 15: 1143-1149.

-Gregory TR (2001): Coincidence, coevolution, or causation? DNA content, cell size, and the Cvalue enigma. Biol Rev Camb Philos Soc 76: 65-101.

Groothuis J, Smid HM (2017): Nasonia parasitic wasps escape from Haller's rule by diphasic, partially isometric brain-body size scaling and selective neuropil adaptations. Submitted.

Hansson BS, Anton S (2000): Function and morphology of the antennal lobe: new developments. Annu Rev Entomol 45:203-231.

-Isler K, Christopher Kirk E, Miller JM, Albrecht GA, Gelvin BR, Martin RD (2008): Endocranial volumes of primate species: scaling analyses using a comprehensive and reliable data set. J Hum Evol 55:967-978.

Libersat F, Pflueger HJ (2004): Monoamines and the orchestration of behavior. Bioscience 54: 17-25.

Niven JE (2016): Neuronal energy consumption: biophysics, efficiency and evolution. Curr Opin Neurobiol 41:129-135.
Niven JE, Farris SM (2012): Miniaturization of nervous systems and neurons. Curr Biol 22:R323-R329.

Perge JA, Niven JE, Mugnaini E, Balasubramanian V, Sterling P (2012): Why do axons differ in caliber? J Neurosci 32:626-638.

Polilov AA (2012): The smallest insects evolve anucleate neurons. Arthropod Struct Dev 41: 29-34.

Polilov AA (2015): Small is beautiful: features of the smallest insects and limits to miniaturization. Annu Rev Entomol 60:103-121.

Polilov AA (2016): Features of the structure of hymenoptera associated with miniaturization: 2. Anatomy of Trichogramma evanescens (Hymenoptera, Trichogrammatidae). Entomol Rev 96:419-431.

Roeder T (1994): Biogenic amines and their receptors in insects. Comp Biochem Physiol C Toxicol Pharmacol 107:1-12.

Salt G (1940): Experimental studies in insect parasitism. VII. The effects of different hosts on the parasite Trichogramma evanescens West. (Hym. Chalcidoidea). Proc R Soc Lond 15: 81-95.

Schindelin J, Arganda-Carreras I, Frise E, Kaynig V, Longair M, Pietzsch T, Preibisch S, Rueden C, Saalfeld S, Schmid B, Tinevez JY, White DJ, Hartenstein V, Eliceiri K, Tomancak P, Cardona A (2012): Fiji: an open-source platform for biological-image analysis. Nat Methods 9: 676-682.

Schmidt JM, Smith JJB (1985): Host volume measurement by the parasitoid wasp Trichogramma minutum: the roles of curvature and surface area. Entomol Exp Appl 39:213-221.

Schmidt JM, Smith JJB (1987): The measurement of exposed host volume by the parasitoid wasp Trichogramma minutum and the effects of wasp size. Can J Zool 65:2837-2845.
Seid MA, Castillo A, Wcislo WT (2011): The allometry of brain miniaturization in ants. Brain Behav Evol 77:5-13.

Seid MA, Goode K, Li C, Traniello JF (2008): Ageand subcaste-related patterns of serotonergic immunoreactivity in the optic lobes of the ant Pheidole dentata. Dev Neurobiol 68:13251333.

Suzuki Y, Tsuji H, Sasakawa M (1984): Sex allocation and effects of superparasitism on secondary sex ratios in the gregarious parasitoid, Trichogramma chilonis (Hymenoptera: Trichogrammatidae). Anim Behav 32:478484.

Van der Woude E, Smid HM (2016): How to escape from Haller's rule: olfactory system complexity in small and large Trichogramma evanescens parasitic wasps. J Comp Neurol 524: 1876-1891.

Van der Woude E, Smid HM (2017): Maximized complexity in miniaturized brains: morphology and distribution of octopaminergic, dopaminergic and serotonergic neurons in Trichogramma evanescens parasitic wasps. Submitted.

-Van der Woude E, Smid HM, Chittka L, Huigens ME (2013): Breaking Haller's rule: brain-body size isometry in a minute parasitic wasp. Brain Behav Evol 81:86-92.

Waage JK, Ming N (1984): The reproductive strategy of a parasitic wasp: I. Optimal progeny and sex allocation in Trichogramma evanescens. J Anim Ecol 53:401-415.

Wehner R, Fukushi T, Isler K (2007): On being small: brain allometry in ants. Brain Behav Evol 69:220-228.

Yamamoto S, Seto ES (2014): Dopamine dynamics and signaling in Drosophila: an overview of genes, drugs and behavioral paradigms. Exp Anim 63:107-119.

Yu DSK, Hagley EAC, Laing JE (1984): Biology of Trichogramma minutum Riley collected from apples in Southern Ontario. Environ Entomol 13:1324-1329. 